PROCEEDINGS OF THE

AMERICAN MATHEMATICAL SOCIETY

Volume 130, Number 8, Pages 2247-2250

S 0002-9939(02)06395-5

Article electronically published on January 23, 2002

\title{
THE DISTRIBUTION OF SEQUENCES IN RESIDUE CLASSES
}

\author{
CHRISTIAN ELSHOLTZ
}

(Communicated by David E. Rohrlich)

\begin{abstract}
We prove that any set of integers $\mathcal{A} \subset[1, x]$ with $|\mathcal{A}| \gg(\log x)^{r}$ lies in at least $\nu_{\mathcal{A}}(p) \gg p^{\frac{r}{r+1}}$ many residue classes modulo most primes $p \ll$ $(\log x)^{r+1}$. (Here $r$ is a positive constant.) This generalizes a result of Erdős and Ram Murty, who proved in connection with Artin's conjecture on primitive roots that the integers below $x$ which are multiplicatively generated by the coprime integers $a_{1}, \ldots, a_{r}$ (i.e. whose counting function is also $\left.c(\log x)^{r}\right)$ lie in at least $p^{\frac{r}{r+1}+\varepsilon(p)}$ residue classes, modulo most small primes $p$, where $\varepsilon(p) \rightarrow$ 0 , as $p \rightarrow \infty$.
\end{abstract}

Let $\operatorname{ord}_{p}(a)$ denote the order of $a$ modulo $p$, where $(a, p)=1$. A quantitative version of Artin's conjecture on primitive roots states that for a fixed integer $a$, not a square, and not -1 , there is a positive proportion of primes such that $\operatorname{ord}_{p}(a)=$ $p-1$. (See [7] for a survey.) In favour of this conjecture, Erdős proved in [2] that for all but $o\left(\frac{y}{\log y}\right)$ of the primes $p \leq y$ one has

$$
\operatorname{ord}_{p}(2)>p^{\frac{1}{2}}
$$

This improved upon the lower bound of $\operatorname{ord}_{p}(2)>p^{\delta}$ for all $\delta<\frac{1}{2}$, proved by Bundschuh; see [1. It is also implicit in section 3 of Hooley's work on Artin's conjecture (see [5]) that for all but $O\left(\frac{y}{(\log y)^{3}}\right)$ primes $p \leq y$ one has $\operatorname{ord}_{p}(a) \gg \frac{\sqrt{p}}{\log p}$. Erdös announced at the end of his paper that the lower bound can be slightly sharpened to

$$
\operatorname{ord}_{p}(2) \geq p^{\frac{1}{2}+\varepsilon(p)},
$$

where $\varepsilon$ is any real function with $\lim _{p \rightarrow \infty} \varepsilon(p)=0$. The details of this were provided by Erdős and Ram Murty in [3]. For related results see also Pappalardi [8].

For a more general situation, they implicitly consider the following. Let $a_{1}, \ldots, a_{r}$ be mutually coprime positive integers and let $p$ be a prime with $\left(p, a_{1} a_{2} \cdots a_{r}\right)=1$. Let $\mathcal{A}_{1}$ be the semigroup of positive integers multiplicatively generated by the $a_{i}$ and let $\mathcal{A}=\mathcal{A}_{1} \cap[1, x]$. Then the elements in $\mathcal{A}$ have the form $a_{1}^{\beta_{1}} a_{2}^{\beta_{2}} \cdots a_{r}^{\beta_{r}} \leq x$, where the $\beta_{i}$ are nonnegative integers. Let $f\left(p, a_{1}, \ldots, a_{r}\right)$ denote the number of distinct residue classes modulo $p$ which are needed to cover all elements of $a \in \mathcal{A}$.

Note that the number of powers of $a$ below $x$ is asymptotically $c_{a} \log x$, and that there are about $c_{a_{1}, \ldots, a_{r}}(\log x)^{r}$ many integers below $x$ which are generated

Received by the editors March 9, 2001.

1991 Mathematics Subject Classification. Primary 11N69, 11N36; Secondary 11B50, 11 A07.

Key words and phrases. Distribution of sequences in residue classes, Gallagher's larger sieve, primitive roots, Artin's conjecture. 
multiplicatively by the $a_{i}$. Here and in the following, the $c$ with various indices stand for positive constants.

In this situation 1 Erdős and Ram Murty prove that, for all but $o\left(\frac{y}{\log y}\right)$ many primes $p \leq y$ with $\left(p, a_{1} \cdots a_{r}\right)=1$ one has

$$
f\left(p, a_{1}, \ldots, a_{r}\right) \geq p^{\frac{r}{r+1}+\varepsilon(p)},
$$

where $\varepsilon$ is an arbitrary real function with $\lim _{p \rightarrow \infty} \varepsilon(p)=0$.

Giving a more quantitative version of this statement one might consider the sequence up to $x$. It is then implicitly understood that $y \ll(\log x)^{r+1}$, since otherwise the sequence $\mathcal{A}$ does not have $\gg y^{\frac{r}{r+1}}$ elements below $x$.

In this note we generalize these results to arbitrary integer sequences. Therefore, the fact that the powers of some element $a$ lie in many residue classes modulo many primes is not necessarily an argument in favour of Artin's conjecture. However, for special sequences like the powers of $a$ the result by Erdős and Ram Murty is stronger by the $\varepsilon(p)$ refinement. We prove the following theorem:

Theorem. Let $x>x_{0}$ and let $\mathcal{A} \subseteq[1, x]$ be a set of positive integers with $|\mathcal{A}| \geq$ $c_{1}(\log x)^{r}$. Let $\nu_{\mathcal{A}}(p)$ denote the number of distinct residue classes modulo $p$ which are necessary to cover $\mathcal{A}$. Let $y=c_{4}(\log x)^{r+1}$. Let

$$
\begin{gathered}
\mathcal{S}=\left\{p \in \mathcal{P} \cap[1, y]: \nu_{\mathcal{A}}(p) \leq c_{2} p^{\frac{r}{r+1}}\right\} \\
\text { and } c_{3}=\frac{|\mathcal{S}|}{\pi(y)} . \text { Let } \beta=\frac{1}{r+1} \text { and } C=\frac{1}{\beta}\left(1-\left(1-c_{3}\right)^{\beta}\right) c_{4}^{\beta} . \text { If } C>c_{2} \text {, then } \\
\frac{c_{2} c_{3} c_{4}}{C-c_{2}} \geq c_{1} \text {. }
\end{gathered}
$$

In typical applications, the counting function $A(x)$, i.e. $c_{1}$ and $r$, might be known. Suppose one wants to make the proportion $c_{3}$ of 'bad primes' very small so that one knows for essentially all primes $p \leq y$, that $\nu_{\mathcal{A}}(p)$ is large. Then one can make an admissible choice of $c_{2}$ and $c_{4}$ as follows: Choose a small $c_{3}$, choose $c_{4} \geq \frac{c_{1}}{c_{3}}$, and put $c_{2}=\frac{C}{2}$. Then trivially $C>c_{2}$ and

$$
\frac{c_{2} c_{3} c_{4}}{C-c_{2}}=\frac{c_{2} c_{3} c_{4}}{c_{2}}=c_{3} c_{4} \geq c_{1}
$$

This implies the following corollary.

Corollary. Let $\mathcal{A}$ be an infinite set of positive integers with counting function $A(x) \gg(\log x)^{r}$. Let $\nu_{\mathcal{A}}(p)$ denote the number of distinct residue classes modulo $p$ which are necessary to cover $\mathcal{A} \cap[1, x]$. Then for all $c_{3}>0$ one can find positive $c_{2}$ and $c_{4}$ such that for all but at most $\frac{c_{3} y}{\log y}$ primes $p \leq y$, where $y=c_{4}(\log x)^{r+1}$, one has $\nu_{\mathcal{A}}(p) \geq c_{2} p^{\frac{r}{r+1}}$.

Unfortunately, it appears, if one allows at most $o\left(\frac{y}{\log y}\right)$ exceptional primes, that is if one requires that $c_{3} \rightarrow 0$ as $x \rightarrow \infty$, then one has to allow that $c_{2}$ and $c_{4}$ vary accordingly.

\footnotetext{
${ }^{1}$ Strictly speaking, their theorem is stated in a more general form for rational numbers. Let us take the opportunity to mention that there is a slight inaccuracy in the description of the $\varepsilon$ in Theorem 4 and part 3 of Theorem 5 of their results (and also in the abstracts in the Math. Reviews and the Zentralblatt): Obviously, one should either replace $p / \varepsilon(p)$ by $p \varepsilon(p)$ or one should take $p / \varepsilon(p)$ with $\lim _{p \rightarrow \infty} \varepsilon(p)=\infty$.
} 
Our main tool is Gallagher's larger sieve, which we state for completeness.

Lemma (Gallagher's larger sieve; see 4]). Let $\mathcal{A} \subseteq[1, x]$ be a set that lies in at most $\nu_{\mathcal{A}}(p)$ residue classes modulo $p$, for $p \in \mathcal{S}$. Then

$$
|\mathcal{A}| \leq \frac{-\log x+\sum_{p \in \mathcal{S}} \log p}{-\log x+\sum_{p \in \mathcal{S}} \frac{\log p}{\nu_{\mathcal{A}}(p)}}
$$

provided the denominator is positive.

Proof of the Theorem. Since we deal with upper bounds and since $\log p$ and $\frac{\log p}{p^{\frac{r}{r+1}}}$ are monotonic functions for $p>p_{0}$, the worst case distribution of the primes in $\mathcal{S}$ is that these primes are as large as possible. If $x$ tends to infinity, then the intervals $[0, c y]$ and $[(1-c) y, y]$ contain asymptotically the same number of primes, $\frac{c y}{\log y}$. The worst case distribution is determined by the primes in $\left[\left(1-c_{3}+o(1)\right) y, y\right]$. For simplicity, we omit $o(1)$ expressions and write $\lesssim$ instead of $\leq$. Moreover, recall that it follows from $\sum_{p \leq z} \log p \sim z$ by partial summation that for $0<\alpha<1$ one has

$$
\sum_{p \leq z} \frac{\log p}{p^{\alpha}} \sim \frac{z^{1-\alpha}}{1-\alpha}
$$

With $\alpha=\frac{r}{r+1}$, so that $1-\alpha=\frac{1}{r+1}=\beta$, we find that

$$
\begin{aligned}
|\mathcal{A}| & \lesssim \frac{-\log x+\sum_{\left(1-c_{3}\right) y \leq p \leq y} \log p}{-\log x+\sum_{\left(1-c_{3}\right) y \leq p \leq y} \frac{\log p}{c_{2} p^{r+1}}} \lesssim \frac{c_{3} y}{-\log x+\frac{1}{c_{2} \beta}\left(y^{\beta}-\left(1-c_{3}\right)^{\beta} y^{\beta}\right)} \\
& =\frac{c_{3} c_{4}(\log x)^{r+1}}{-\log x+\frac{C}{c_{2}} \log x}=\frac{c_{2} c_{3} c_{4}}{C-c_{2}}(\log x)^{r}
\end{aligned}
$$

Suppose that we have $C>c_{2}$ but $\frac{c_{2} c_{3} c_{4}}{C-c_{2}}<c_{1}$. This is, for sufficiently large $x$, a contradiction to our assumption $|\mathcal{A}| \geq c_{1}(\log x)^{r}$.

Remark. Matthews (see [6]) considered questions related to that of Erdös and Ram Murty in a more general context of algebraic groups and abelian varieties. For the classical case of Artin's conjecture he proved that for almost all primes and for all positive $\varepsilon$ one has $\nu(p)>p^{\frac{1}{2}-\varepsilon}$. (Apparently he was unaware of [1] and [2].) He mentions further applications to nilpotent groups and to manifolds due to Milnor, Tits, and Wolf.

\section{REFERENCES}

[1] Bundschuh, P., Solution of problem 618. Elemente der Mathematik 26 (1971), 43-44.

[2] Erdős, P., Bemerkungen zu einer Aufgabe in den Elementen. Arch. Math. 27 (1976), 159-163. MR 53:7969

[3] Erdős, P.; Murty, M. Ram, On the order of $a(\bmod p)$. Number theory (Ottawa, 1996), 87-97, CRM Proc. Lecture Notes, 19. MR 2000c: 11152

[4] Gallagher, P.X., A larger sieve. Acta Arith. 18 (1971), 77-81. MR 45:214

[5] Hooley, C., On Artin's conjecture. J. Reine Angew. Math. 225 (1967), 209-220. MR 34:7445

[6] Matthews, C.R., Counting points modulo $p$ for some finitely generated subgroups of algebraic groups. Bull. London Math. Soc. 14 (1982), 149-154. MR 83c:10067 
[7] Murty, M. Ram, Artin's conjecture for primitive roots. Math. Intelligencer 10 (1988), 59-67. MR 89k:11085

[8] Pappalardi, F., On the order of finitely generated subgroups of $Q^{*}(\bmod p)$ and divisors of $p-1$. J. Number Theory 57 (1996), 207-222. MR 97d:11141

Institut für Mathematik, Technische Universität Clausthal, Erzstrasse 1, D-38678 Clausthal-Zellerfeld, Germany

E-mail address: elsholtz@math.tu-clausthal.de 